\title{
Dependence of chain conformation on degree of sulfonation and counterion dissociation of sodium poly(styrene sulfonate) in semidilute aqueous solution
}

\author{
King-Fu Lin*, Horng-Long Cheng, Yu-Hui Cheng \\ Department of Materials Science and Engineering, National Taiwan University, Taipei 10617, Taiwan, ROC
}

Received 2 July 2003; received in revised form 23 November 2003; accepted 30 January 2004

\begin{abstract}
The chain conformation of sodium poly(styrenesulfonate) (NaPSS) in the semidilute aqueous solution, estimated from the plots of reduced viscosity versus segmental concentration, was found to be dependent on the degree of sulfonation and dissociation extent of counterions. The latter, altered by the content of sodium chloride external salt, was estimated from the ionic conductivity measurements on the basis of the modified Manning's conductivity theories with consideration of the influence of chain overlapping in the semidilute regime. The results indicate that the increase of chain extension of NaPSS with counterion dissociation was further enhanced by increasing the degree of sulfonation.
\end{abstract}

(C) 2004 Elsevier Ltd. All rights reserved.

Keywords: Polyelectrolyte; Sodium poly(styrenesulfonate); Chain conformation

\section{Introduction}

In more than a half century ago, Fuoss and Strauss [1] reported that the polyelectrolyte solutions show an anomalous behavior in semidilute salt-free systems or with low salt content, in that the reduced viscosity $\eta_{\text {red }}$ is abruptly increased with decreasing the segmental concentration $c_{\mathrm{p}}$. They described it with an empirical equation: $\eta_{\text {red }}=$ $A /\left(1+B c_{\mathrm{p}}^{1 / 2}\right)$, where $A$ and $B$ are constants. In 1976, de Gennes et al. [2] derived a similar expression $\eta_{\text {red }} \sim c_{\mathrm{p}}^{-1 / 2}$, by assuming that the polyelectrolyte chains were in a rod form. However, most of the experimental data [3-5] did not support the relation $\eta_{\text {red }} \sim c_{\mathrm{p}}^{-1 / 2}$. Recently, we have suggested the polyelectrolytes as a flexible chain with a succession of segments (or blobs) each carrying a $g$-number of monomers [6]. By referring to Rouse dynamics and neglecting the entanglement effects, the viscosity of the polyelectrolyte solutions, $\eta$ was expressed as follows [7]

$\eta-\eta_{0}=\frac{1}{36} \rho \bar{r}_{0}^{2} N^{*} f_{0}$

\footnotetext{
* Corresponding author. Tel.: + 886-223-928-290; fax: + 886-223-634562.

E-mail address: kflin@ccms.ntu.edu.tw (K.F. Lin).
}

where $\eta_{0}$ is the viscosity of solvent, $\rho$, the moles of polymer chains/volume $\approx c_{\mathrm{p}} / N, N$, the number of segments per chain, $\bar{r}_{0}^{2}=N^{*} \xi^{2}$, the mean square end-to-end distance of a chain, $N^{*}=N / g$, the number of blobs per chain, $f_{0}=$ $3 \pi \eta_{0} \xi$, the friction coefficient for one blob (here we assume the system is undraining inside the blob) against the solvent, and $g \approx c_{\mathrm{p}} \xi^{3}$, indicating that each volume $\xi^{3}$ is occupied by one blob on the average. Thus, Eq. (1) was rewritten as follows [6]

$$
\eta_{\mathrm{red}}=\frac{\eta-\eta_{0}}{\eta_{0} c_{\mathrm{p}}} \approx \frac{N}{c_{\mathrm{p}}^{2} \xi^{3}}
$$

Based on de Gennes et al. [2], the crossover from the dilute to the semidilute regime is characterized by a critical concentration $c^{*} \approx N / R^{3}$, where $R$ is the average end to end distance of polyelectrolyte chains. Due to the overlapping of the polymer chains and the formation of an isotropic network in the semidilute regime, all properties of the polymer solutions lose their molecular mass dependence. Thus, the behavior of the polymer solution is governed by the correlation length $\xi\left(c_{\mathrm{p}}\right)$, which is molecular weight independence. We then assumed $R=N^{\nu} a$, in which $a$ is the length per mer and $\nu$ is the chain conformation index in a range of $0.5 \leq \nu \leq 1$, corresponding to the chain 
conformation from Gaussian flexible to rigid rod extreme. By using the formula of correlation length [2]

$$
\xi=R\left(\frac{c^{*}}{c_{\mathrm{p}}}\right)^{m} \quad\left(c_{\mathrm{p}} \gg c^{*}\right)
$$

where $m$ is the unknown power of the concentration, we obtained $\xi \approx N^{\nu+m-3 \nu m} c_{\mathrm{p}}^{-m}$. Since $\xi \approx N^{0}, m=\nu /(3 \nu-1)$. From Eq. (2) and $\eta_{\text {red }} \approx c_{\mathrm{p}}^{n}$ expression, we obtained [6]

$\nu=\frac{n+2}{3 n+3}$

It can be found that the exponent $n$ changes from $-1 / 2$ for a rod-like chain conformation $(\nu=1)$ to 1 for a Gaussian flexible chain $(\nu=0.5)$. For a real free-coil chain $(\nu=0.6)$, $n=0.25$ was obtained. A similar result was also obtained by Muthukumar [8], who give $n=0.25$ for the polyelectrolyte solutions with high salt content.

The above new scaling relation was able to be employed to estimate the chain conformations of polyelectrolytes in the semidilute regime with and without containing external salts, simply by calculating $n$ from the double-logarithmic plots of reduced viscosity versus concentration [6]. It is noteworthy that the conformation of polyelectrolyte chains is mainly influenced by their charge density and counterion dissociation. Although the conductivity of polyelectrolyte solution can provide some measure of counterion dissociation [9], the interpretation for the conductivity measurements of polyelectrolyte solutions has relied primarily on the Manning's conductivity theories [10,11]. However, it is still in doubt if they are able to apply to the semidilute regime because of overlapping of the polymer chains. Kuhn et al. [12] has suggested that Mannings' theory underestimates the number of condensed counterions in the semidilute regime. We have also found that the extent of aggregation of polyelectrolytes in the semidilute salt-free aqueous solution substantially affects the ionic conductivity of the solution [13].

In this study, sodium poly(styrenesulfonate) (NaPSS) with various degrees of sulfonation (or charge densities) were prepared and estimated their chain conformations in semidilute salt-free and salt-containing aqueous solutions, by using the double-logarithmic plots of reduced viscosity versus segmental concentrations. Their dissociation extent of counterion was estimated on the basis of the Manning's conductivity theories with some modification by considering the influence of chain overlapping in the semidilute regime [14,15]. Thus, the dependence of chain conformation on the degree of sulfonation and counterion dissociation of NaPSS in the semidilute aqueous solutions was established.

\section{Experimental}

\subsection{Materials}

A narrow molecular weight polystyrene (PS) sample (purchased from Aldrich Chemical Company), which had a number average molecular weight of 180,000 with a $M_{\mathrm{w}} / M_{\mathrm{n}}$ ratio of 1.1, was used to prepare NaPSS with various degrees of sulfonation. The preparation procedure followed the method provided by Vink [16] with some modifications. In general, $0.25 \mathrm{~g}$ PS was dissolved in $70 \mathrm{ml}$ cyclohexane first, and then transferred to the flask that has already been loaded with $50 \mathrm{ml}$ sulfuric acid (40 and $25 \mathrm{ml}$ were also used to vary the degrees of sulfonation) and $5 \mathrm{~g} \mathrm{P}_{2} \mathrm{O}_{5}$ (98.0\%, Showa Co. Japan). After stirred for $80 \mathrm{~min}$ at $55^{\circ} \mathrm{C}$, the reaction mixture became separated into pure cyclohexane and a faintly yellow phase containing the mineral acids and poly(styrenesulfonic acid) (PSSA). Then it was allowed to stand for $70 \mathrm{~min}$ before cooled to $0{ }^{\circ} \mathrm{C}$. After that, $20 \mathrm{~g}$ crushed ice was slowly added to the flask with stirring. The mixture was then transferred to a separating funnel and the heavier mineral acids were separated from the PSSA/ cyclohexane mixture. $120 \mathrm{ml}$ water (ultrapure, $18.3 \mathrm{M} \Omega$ ), which was purified by the Barnstead Easypure RF system, was added to the latter to dissolve PSSA. After separated from cyclohexane, the PSSA solution was filtered through a porous glass filter and dialyzed in a Sprectra/Pro regenerated cellulose membrane tube (molecular weight cutoff at 3500) against ultrapure water. The dialysis tube was cleaned by using the Sprectra/Pro sulfide removal kit. The dialysis was completed after the conductivity of the repeatedly refreshed water surrounding the dialysis tube closed to $1 \mu \mathrm{S} / \mathrm{cm}$. Afterwards, the PSSA solution was freeze-dried to a solid product $(\sim 0.5 \mathrm{~g})$, which retained up to $10 \mathrm{wt} \%$ water as measured by thermogravimetric analyzer (Du Pont model 9900-954 TGA). The obtained PSSA was dissolved in $250 \mathrm{ml}$ ultrapure water and then converted to the NaPSS solution by potentiometric titration with $0.1 \mathrm{M} \mathrm{NaOH}$. After that, it was dialyzed again to remove the excess $\mathrm{NaOH}$. The degree of sulfonation $(\alpha)$ and average segmental equivalent weight $\left(m_{\mathrm{s}}\right)$ (molecular weight divided by number of sulfonate groups in a chain) of prepared NaPSS were determined from the amount of $\mathrm{NaOH}$ consumed in titration. The results were listed in Table 1. The molecular weight and molecular weight distribution of the prepared NaPSS were measured by gel permeation chromatography (Testhigh Series III RI detector with $\mathrm{H}_{2} \mathrm{O} / \mathrm{CH}_{3} \mathrm{OH} / \mathrm{NaNO}_{3} /$ $\mathrm{NaH}_{2} \mathrm{PO}_{4}$ buffer solution as a mobile phase). The calibration

Table 1

Data for the prepared NaPSS samples

\begin{tabular}{lrlll}
\hline Sample & $\alpha(\%)$ & $m_{\mathrm{s}}\left(\mathrm{g} \mathrm{mol}^{-1}\right)$ & $M_{\mathrm{n}}\left(\mathrm{g} \mathrm{mol}^{-1}\right)$ & $M_{\mathrm{w}} / M_{\mathrm{n}}$ \\
\hline 1 & $\sim 100$ & 206 & 350,000 & 1.10 \\
2 & 83 & 227.3 & 330,000 & 1.11 \\
3 & 51 & 305.9 & 270,000 & 1.08 \\
\hline
\end{tabular}


curve was obtained from the monodispersed poly(ethylene oxide) standard sample.

The NaPSS aqueous solutions with various degrees of sulfonation were prepared from the volumetric dilutions for concentrations ranging from 0.015 to $0.00002 \mathrm{~g} / \mathrm{dl}$, respectively. Polymer solutions with added sodium chloride $(\mathrm{NaCl})$ salts were prepared from the highest polymer concentration first and then gradually diluted with sodium chloride aqueous solutions having predetermined concentrations.

\subsection{Methods}

The viscosity measurements were carried out by using a capillary Ubbelohde viscometer (type 0A, Cannon co.) immersed in a thermostatic water bath (TAMSON co., model TV 2000) maintained at $30 \pm 0.01{ }^{\circ} \mathrm{C}$. A typical flow time of polymer solutions in a viscometer was measured in a range of 100-300 s with an accuracy of $\pm 0.1 \mathrm{~s}$, and each flow time was determined by repeating at least five time measurements. The conductivity measurements were conducted in a beaker containing $100 \mathrm{ml}$ polymer solutions at $30 \pm 0.01{ }^{\circ} \mathrm{C}$ under a nitrogen atmosphere, by using a conductivity cell, with platinized electrodes and a cell constant of $3.64 \mathrm{~cm}^{-1}$. The signal was recorded on a Radiometer Copenhagen CDC-230 conductivity meter, and the data were then subtracted the conductivities of water and $\mathrm{NaCl}$ external salts.

\section{Results and discussion}

\subsection{Salt-free polyelectrolyte solutions}

Fig. 1 shows the double-logarithmic plots of reduced viscosity $\eta_{\text {red }}$ versus segmental concentration $c_{\mathrm{p}}$ of NaPSS with various degrees of sulfonation in salt-free aqueous solutions. Two regimes separated by $c_{\mathrm{p}, \max }$, that shows the

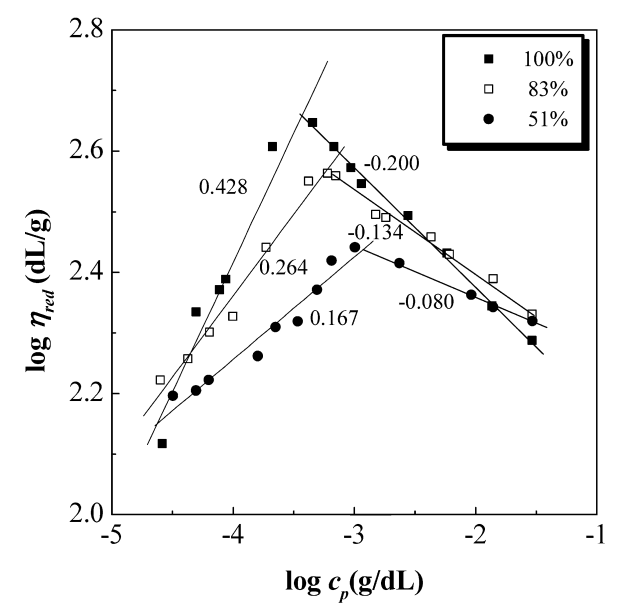

Fig. 1. Double-logarithmic plots of $\eta_{\text {red }}$ versus $c_{\mathrm{p}}$ of NaPSS with various degrees of sulfonation in salt-free aqueous solutions. The numbers obtained from the slops denote the exponent $n$ in $\eta_{\text {red }} \sim c_{\mathrm{p}}^{n}$. highest $\eta_{\text {red }}$, were clearly seen. It is convenient to assign $c_{\mathrm{p}, \max }$ as a break point that separates the dilute and semidilute regimes. According to Muthukumar, [8] the behavior that $\eta_{\text {red }}$ increases with $c_{\mathrm{p}}$, in the dilute solution can be described by the Zimm dynamics. In this regime, the chains barely interact with each other so that their properties depend on the molecular weight of polymers. When the polyelectrolyte concentration was increased to the semidilute regime, the polymer chains began to overlap. Thus, their chains have a tendency to aggregate together and melt into an isotropic lattice, which is often pictured as a collection of uncorrelated blobs with average diameter much less than the end-to-end distance of polyelectrolyte chains.

According to our derived scaling relation, [6] the average end-to-end distance $R=N^{\nu} a$ of NaPSS in the semidilute regime can be estimated by Eq. (4), i.e., $\nu=$ $(n+2) / 3(n+1)$, where $n$ in $\eta_{\text {red }} \sim c_{\mathrm{p}}^{n}$ was obtained from the slope of Fig. 1. The obtained chain conformation indexes of NaPSS with various degrees of sulfonation were listed in Table 2. Apparently, with increasing the degree of sulfonation, the chain was more extended.

Fig. 2 shows the plots of the equivalent conductivities of NaPSS with various degrees of sulfonation in aqueous solutions versus the segmental concentration $c_{\mathrm{p}}$ in monomole/l. The equivalent conductivities barely changed with the concentration in a range of $0.0022>c_{\mathrm{p}}>0.00015$ monomole/l, which is in the semidilute regime by referring to Fig. 1 . Thus, we compared the experimental data of equivalent conductivity $\Lambda$ with the theoretical prediction by the Manning's conductivity theory for salt-free polyelectrolyte solutions [10].

$\Lambda=f_{\text {theo }}\left(\lambda_{\mathrm{c}}^{0}+\lambda_{\mathrm{p}}\right)$

where

$f_{\text {theo }}=0.886\left|z_{\mathrm{c}}\right|^{-1} \xi_{\mathrm{M}}^{-1}, \quad \xi_{\mathrm{M}}=\frac{Q}{b}=\frac{e^{2}}{\varepsilon K T b}>\left|z_{\mathrm{c}}\right|^{-1}(6)$

$\lambda_{\mathrm{c}}^{0}$ is the equivalent conductivity of the counterion in pure solvent, $z_{\mathrm{c}}$ is the valence of the counterion, $\xi_{\mathrm{M}}$ is the charge density parameter, $Q=e^{2} / \varepsilon K T$ is the Bjerrum length ( $e$ is the electronic charge and $\varepsilon$ is the dielectric constant of water), $b$ is the charge spacing of the polyelectrolyte chain, and $\lambda_{\mathrm{p}}$ the equivalent conductivity of the polyions in the

Table 2

Solution properties of NaPSS samples in the salt-free semidilute aqueous solutions

\begin{tabular}{llll}
\hline Sample & $\nu^{\mathrm{a}}$ in $R=N^{\nu} a$ & $f_{\text {theo }}$ & $f$ \\
\hline 1 & 0.750 & 0.308 & 0.193 \\
2 & 0.718 & 0.371 & 0.214 \\
3 & 0.696 & 0.604 & 0.223 \\
\hline
\end{tabular}

${ }^{a}$ Chain conformation index was estimated from Fig. 1 in the semidilute regime. 


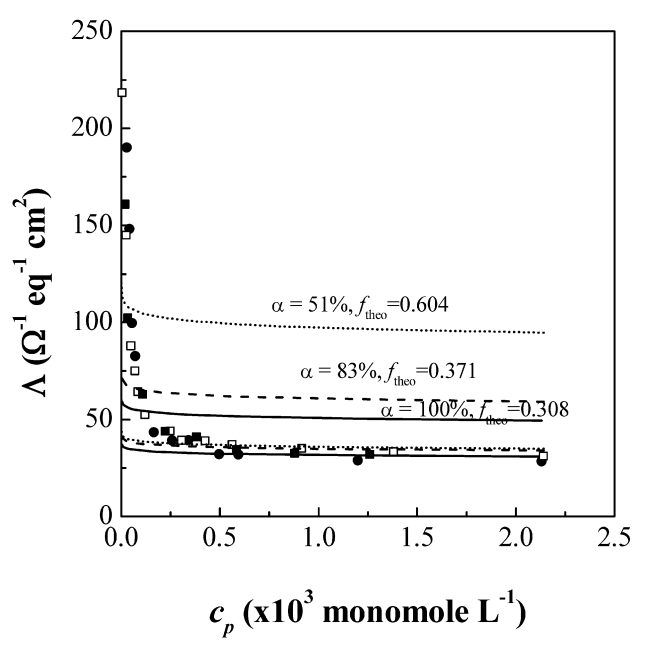

Fig. 2. Concentration dependence of equivalent conductivities for the saltfree aqueous solutions of NaPSS with following degrees of sulfonation: ( $\square$ ) $100 \%$, ( $\square) 83 \%$, and (๑) $51 \%$. Note: The curves above the data points were drawn from Eq. (5) with $f_{\text {theo }}$, whereas those on the data points were drawn with adjusted $f$ to substitute $f_{\text {theo }}$ in Eq. (5).

solution, given by

$\lambda_{\mathrm{p}}=\frac{279 A\left|z_{\mathrm{c}}\right|^{-1} \ln \kappa r}{1+43.2\left(\left|z_{\mathrm{c}}\right| \lambda_{\mathrm{c}}^{0}\right)^{-1} A \ln \kappa r}$

where

$A=\frac{\varepsilon K T}{3 \pi \eta e}$

$\kappa$ is the Debye-Hückel screening constant

$\kappa^{2}=\left(\frac{4 \pi e^{2}}{\varepsilon K T}\right) \frac{c_{\mathrm{p}}}{\xi_{\mathrm{M}}}$

and $r$ is the radius of the polyions. Eq. (6) indicates that $f_{\text {theo }}$ only depends on the charge densities and is not a function of the concentration, whereas $\Lambda$ obtained from Eq. (5) slightly depends on the segmental concentration because of $\lambda_{\mathrm{p}}$. We have used Eqs. (5)-(9) to calculate $f_{\text {theo }}$ and $\Lambda$ for the NaPSS solutions with various degrees of sulfonation, the results of which were listed in Table 2 and plotted in Fig. 2, respectively. The discrepancy between the Manning's conductivity theory and the experimental results in the semidilute regime for $c_{\mathrm{p}}>0.00015$ monomole/l is due to the fact that the former does not consider the overlapping of the polymer chains. As a result, the fraction of uncondensed counterions should be less than that predicted by Eq. (6).

If we allow the fraction of uncondensed counterions in Eq. (6) to be adjustable (i.e. $f_{\text {theo }}$ is replaced by $f$ in order to fit the experimental data), we found that the predicted equivalent conductivities are well matched with the experimental data in the region of $c_{\mathrm{p}}>0.00015$ monomole/l as shown in Fig. 2. The obtained $f$ was also listed in Table 2. Obviously, the Manning's condensation theory underestimates the number of condensed counterions in the semidilute regime. Similar result was also reported for the poly(xylylene tetrahydrothiophenium chloride) (PXT) in the semidilute aqueous solution [14]. The deviation of $f$ from $f_{\text {theo }}$ was considered as the influence of overlapping of polyelectrolyte chains on the counterion condensation. The results also indicate that the lower the degree of sulfonation or charge density, the counterion dissociation is greater, which is apparently attributed to the lower coulomb attractive force created by the dissociation of counterions. Briefly, it is interesting to note from Table 2 that the higher the degree of sulfonation, NaPSS chain is more extended but the dissociation extent of counterions is less.

\subsection{Salt-containing polyelectrolyte solutions}

When NaPSS aqueous solutions were added with $\mathrm{NaCl}$ salts, $c_{\mathrm{p}, \max }$ shifted to higher segmental concentration with smaller $\eta_{\text {red }}$ but larger $n$ in $\eta_{\text {red }} \sim c_{\mathrm{p}}^{n}$ as shown in Fig. 3 . With increase of $\mathrm{NaCl}$ concentration $c_{\mathrm{s}}$ from $2 \times 10^{-6}$ to $5 \times 10^{-4} \mathrm{M}, c_{\mathrm{p}, \max }$ continuously shifted to higher concentration. At $c_{\mathrm{s}}=5 \times 10^{-4} \mathrm{M}, c_{\mathrm{p} \text {,max }}$ became indiscernible for NaPSS with the smallest degree of sulfonation $\alpha=51 \%$. With further increase of $c_{\mathrm{s}}$ to $1 \times 10^{-3} \mathrm{M}, c_{\mathrm{p}, \max }$ was not observable for all three degrees of sulfonation. The increase of $n$ in $\eta_{\text {red }} \sim c_{\mathrm{p}}^{n}$ with $c_{\mathrm{s}}$ indicates that the conformation of polyelectrolyte chains turned to a more coiled state. The chain conformation indexes of NaPSS estimated by Eq. (4) with various degrees of sulfonation and salt contents were listed in Table 3. To correlate the chain conformation of NaPSS with the dissociation extent of counterions in the salt-containing aqueous solutions, the latter was estimated from the ionic conductivity measurements on the basis of another Manning's conductivity theory derived particularly for the polyelectrolyte solutions with external salts [11].

Fig. 4 shows that the equivalent conductivities of NaPSS in the region of $0.0022>c_{\mathrm{p}}>0.00015$ monomole/l were decreased with $c_{\mathrm{s}}$ for all three NaPSS samples with various degrees of sulfonation. For the salt-containing polyelectrolyte solutions, Manning [11] has modified $\lambda_{\mathrm{p}}$ as following $\lambda_{\mathrm{p}}=\mathfrak{J} U$

where $\mathfrak{I}$ is the Faraday constant and $U$ is the electrophoretic mobility of polyelectrolyte chains, expressed by

$300 U=\left(300 U^{*}\right)(\alpha / \beta)$

Table 3

The chain conformation index $\nu$ and dissociation extent $f$ of counterions of NaPSS with various degrees of sulfonation in the semidilute aqueous solution containing various $\mathrm{NaCl}$ concentrations

\begin{tabular}{|c|c|c|c|c|c|c|}
\hline \multirow[t]{2}{*}{ Salt contents (M) } & \multicolumn{2}{|c|}{$\alpha=100 \%$} & \multicolumn{2}{|c|}{$\alpha=83 \%$} & \multicolumn{2}{|c|}{$\alpha=51 \%$} \\
\hline & $\nu$ & $f$ & $\nu$ & $f$ & $\nu$ & $f$ \\
\hline $2 \times 10^{-6}$ & 0.723 & 0.175 & 0.702 & 0.190 & 0.690 & 0.220 \\
\hline $2 \times 10^{-5}$ & 0.698 & 0.152 & 0.679 & 0.184 & 0.679 & 0.215 \\
\hline $5 \times 10^{-4}$ & 0.684 & 0.115 & 0.681 & 0.136 & 0.661 & 0.155 \\
\hline $1 \times 10^{-3}$ & 0.654 & 0.097 & 0.659 & 0.108 & 0.661 & 0.093 \\
\hline
\end{tabular}




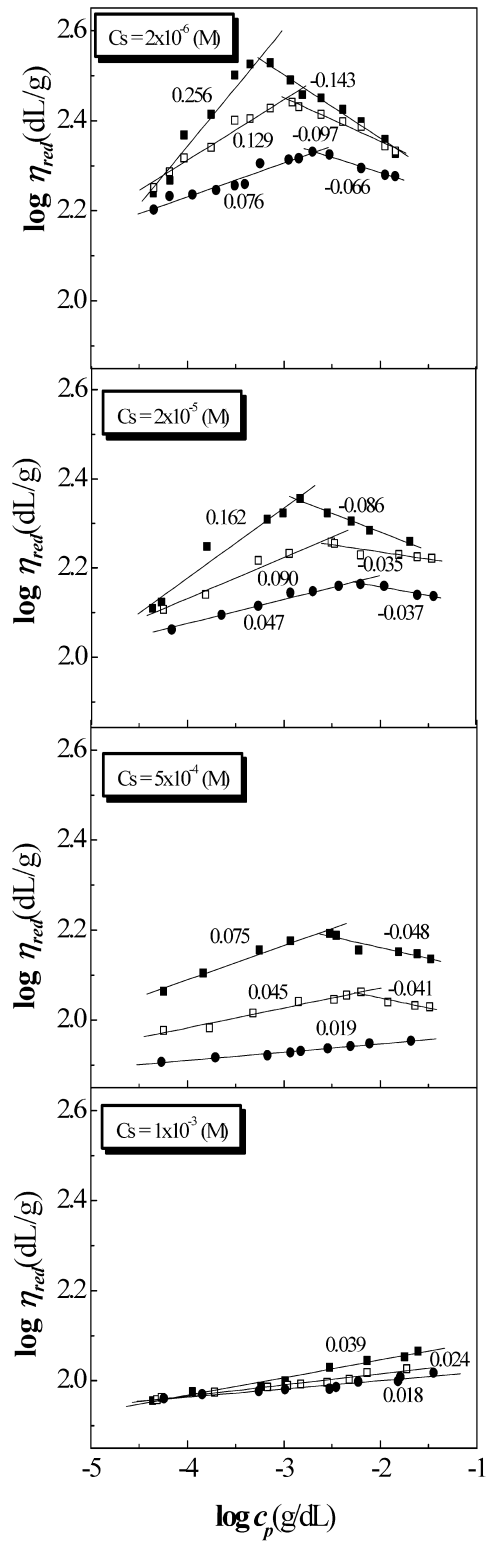

Fig. 3. Double-logarithmic plots of $\eta_{\text {red }}$ versus $c_{\mathrm{p}}$ of NaPSS with (ם) $100 \%$, ( $\square$ ) $83 \%$, and $(\bullet)$ degrees of sulfonation in aqueous solutions with indicated concentration of $\mathrm{NaCl}$. The numbers obtained from the slops denote the exponent $n$ in $\eta_{\text {red }} \sim c_{\mathrm{p}}^{n}$.

where $300 U^{*}$ contains only the charged-solvent effect and was given by

$300 U^{*}=\left|z_{1}\right|^{-1}[\varepsilon k T /(3 \pi \eta e)]|\ln (k b)|$

while

$\alpha=1-\frac{1}{3} \nu_{1}\left(\nu_{1}+\nu_{2}\right)^{-1}\left|z_{1} z_{2}\right|^{-1}\left(z_{1}^{2}-z_{2}^{2}\right)$

$\beta=1+108 \nu_{1}\left(\nu_{1}+\nu_{2}\right)^{-1}\left(300 U^{*}\right)\left(\frac{z_{1}^{2}}{\lambda_{1}^{\mathrm{s}}}+\frac{z_{2}^{2}}{\lambda_{2}^{\mathrm{s}}}\right)$

where $\nu_{i}$ is the number of ions of species $i$ in the formula for the small electrolyte (e.g. $\nu_{1}=1, \nu_{2}=1$ for $\mathrm{NaCl}$ ); $\lambda_{i}^{\mathrm{s}}$ is the ionic conductivity of the small ions; $z_{i}$ is the valence of

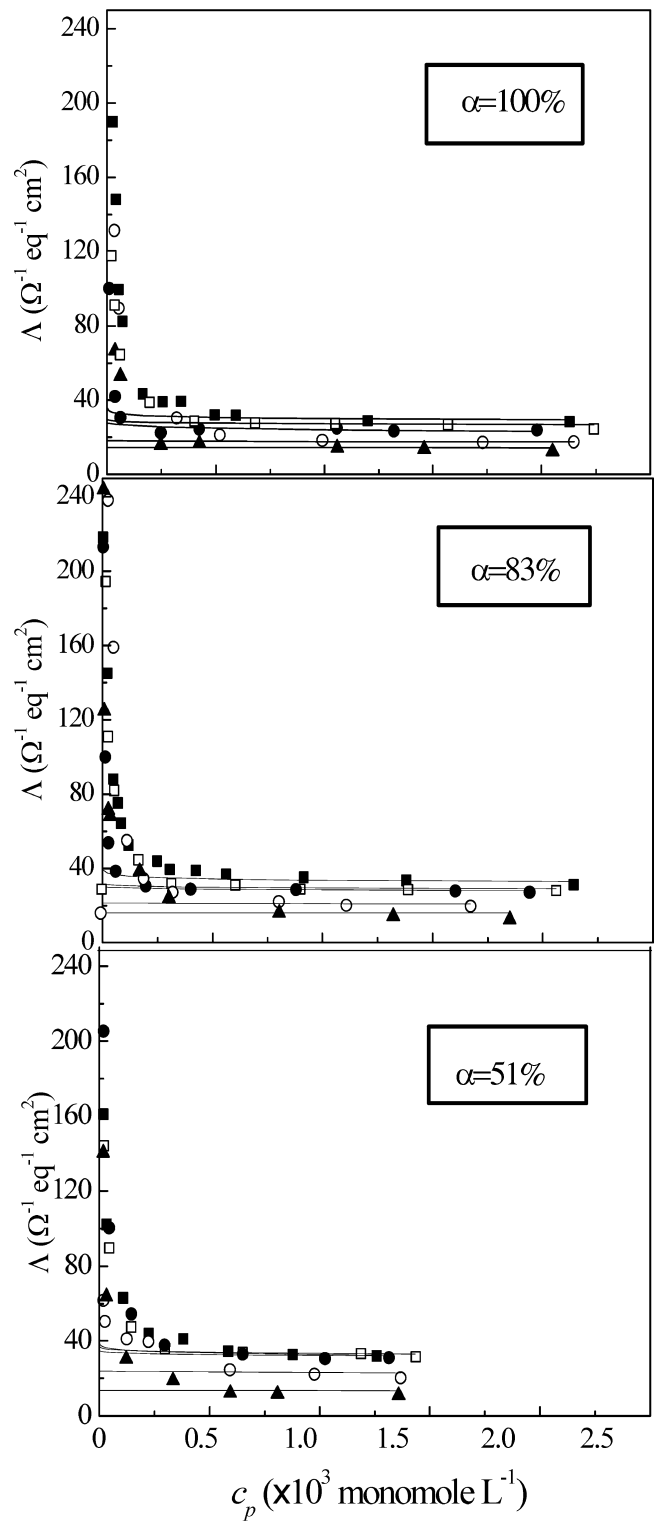

Fig. 4. Concentration dependence of equivalent conductivities of NaPSS with indicated degrees of sulfonation in aqueous solutions with following $\mathrm{NaCl}$ concentrations $c_{\mathrm{s}}:(\boldsymbol{\square})$ salt-free, $(\square) 2 \times 10^{-6} \mathrm{M},(\bullet) 2 \times 10^{-5} \mathrm{M}$, (O) $5 \times 10^{-4} \mathrm{M}$, and (A) $1 \times 10^{-3} \mathrm{M}$.

the small ions; and $\kappa$ defined here is as follows:

$\kappa^{2}=\left(\frac{4 \pi e^{2}}{\varepsilon K T}\right)\left(\frac{c_{\mathrm{p}}}{\xi_{\mathrm{M}}}+2 c_{\mathrm{s}}\right)$

Eqs. (10)-(15) were used to calculate $\lambda_{\mathrm{p}}$ of NaPSS polyions as functions of $c_{\mathrm{p}}$ and $c_{\mathrm{s}}$ for various degrees of sulfonation. $f$ was then obtained by fitting the experimental data in Fig. 4 with calculated $\lambda_{\mathrm{p}}$ substituting for Eq. (5) and allowing $f_{\text {theo }}$ replaced by the adjustable $f$. The results shown in Table 3 indicate that $f$ decreased with the concentration of external salts for all three NaPSS samples. Since $f$ is also an indication of chain conformation for NaPSS in the semidilute regime, we plotted $\nu$ obtained from Figs. 1 and 3 as a function of $f$ obtained from Figs. 2 and 4. The results 


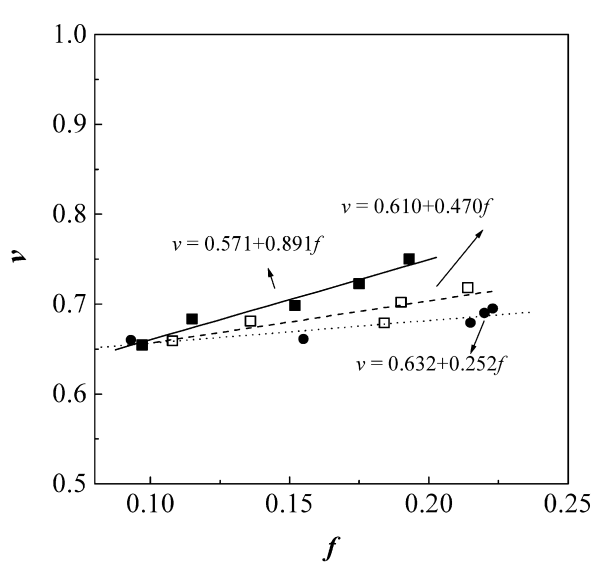

Fig. 5. Plots of the chain conformation index $\nu$ versus the dissociation extent $f$ of counterions in salt-containing aqueous solutions of NaPSS with

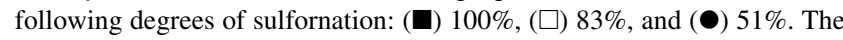
lines were obtained by fitting the data with the first-order least-square method.

shown in Fig. 5 indicate that they could be fit by the firstorder least-square method with following relationship:

$\nu=\nu_{0}+B f$

where $\nu_{0}$ is the chain conformation index at $f \rightarrow 0$ and equal to $0.604 \pm 0.02$ averaged from the data of three NaPSS samples with various degrees of sulfonation; and $B$ is the proposition constant between $\nu$ and $f$. The increase of $B$ with the degree of sulfonation shown in Fig. 5 indicates that the trend of increasing the chain extension of polyelectrolytes with the extent of counterion dissociation was enhanced by increasing the charge density.

When NaPSS approached to a neutral chain under the condition of $f \rightarrow 0$ or $B \rightarrow 0$ (at $\alpha \rightarrow 0$ ), $\nu$ closed to 0.6 , which is agreeable with most of the theoretical predictions [8,17]. Muthukumar [8] gave $n=0.25$ in $\eta_{\text {red }} \sim c_{\mathrm{p}}^{n}$ for the polyelectrolyte solutions with high salt content, which is corresponding to $\nu=0.6$ from Eq. (4). Rubinstein et al. [17] have pointed out that, for the polyelectrolyte solutions in the semidilute regime with high salt content to screen the Coulomb interaction between charged monomers, the concentration dependence of the correlation length $\xi$ (defined in Eq. (3)) is similar to that of the uncharged polymer $\xi \sim c_{\mathrm{p}}^{-0.75}$, which is corresponding to $\nu=0.6$ according to our scaling relation.

\section{Conclusions}

The relationship between the chain conformation of NaPSS with various degrees of sulfonation and the dissociation extent of counterions in the semidilute region was established in this study. For the salt-free system, to increase the degree of sulfonation increases the chain conformation index $\nu$ but decreases the dissociation extent $f$ of counterions. For the salt-containing systems, $\nu$ increased linearly with $f$ by decreasing the salt content and the slope was increased with the degree of sulfonation. All the estimated $\nu$ approached to the theoretical value of 0.6 for neutral chain when the dissociation extent of counterions was linearly extrapolated to zero.

\section{Acknowledgements}

The authors acknowledge the financial support of the National Science Council in Taiwan, Republic of China, through Grant NSC 88-2216-E002-01.

\section{References}

[1] Fuoss RM, Strauss UP. J Polym Sci 1948;3(2):246-63.

[2] De Gennes PG, Pincus P, Velasco RM, Brochard F. J Phys (Paris) 1976;37:1461-73.

[3] Yamanaka J, Araie H, Matsuoka H, Kitano H, Ise N, Yamaguchi T, Saeki S, Tsubokawa M. Macromolecules 1991;24:6156-9.

[4] Roure I, Rinaudo M, Milas M. Ber Bunsen-Ges Phys Chem 1996; 100(6):703-6.

[5] Cohen J, Priel Z, Rabin Y. J Chem Phys 1988;88(11):7111-6.

[6] Lin KF, Cheng HL. Macromolecules 2000;33:4961-5.

[7] Bueche F. Physical properties of polymer. New York: Interscience; 1970. Chapter 3.

[8] Muthukumar M. J Chem Phys 1997;107:2619-35.

[9] Colby RH, Boris DC, Krause WE, Tan JS. J Polym Sci Polym Phys 1997;35:2951-60.

[10] Manning GS. J Phys Chem 1975;79(3):262-8.

[11] Manning GS. J Phys Chem 1981;85(11):1506-15.

[12] Kuhn PS, Levin Y, Barbosa MC. Macromolecules 1998;31:8347-55.

[13] Lin KF, Yang SN, Cheng HL, Cheng YH. Macromolecules 1999;32: 4602-7.

[14] Cheng HL, Lin KF. Langmuir 2002;18:7287-90.

[15] Cheng HL, Lin KF. Macromolecules 2003;36:6949-51.

[16] Vink H. Makromol Chem 1981;182:279-81.

[17] Dobrynin AV, Colby RH, Rubinstein M. Macromolecules 1995;28: 1859-71. 